\title{
A Refined Approximation of a Finite Capacity Polling System under ATM Bursty Arrivals
}

Y. Frank Jou

Arne A. Nilsson

Fuyung Lai

Center for Communications and Signal Processing Department of Electrical and Computer Engineering North Carolina State University

\section{TR-92/16}

September 1992 


\title{
A Refined Approximation of a Finite Capacity Polling System under ATM Bursty Arrivals
}

\author{
Y. Frank Jou and Arne A. Nilsson \\ Center for Communications and Signal Processing \\ Department of Electrical and Computer Engineering \\ North Carolina State University \\ Raleigh, N.C. 27695-7914 \\ Fuyung Lai \\ IBM \\ V57/B660 P.O. Box 12195 \\ Research Triangle Park, N.C. 27709
}

\begin{abstract}
This paper is concerned with both the mean delay and the probability of cell loss that bursty arrivals incur in an ATM switching system which can be modeled as a finite capacity polling system with nonexhaustive cyclic service. The arrival process to each input port of the system is assumed to be bursty and is modeled by an Interrupted Bernoulli Process(IBP). A practical polling system with finite capacity, as the one we deal with here, does not lend itself to an exact solution. In this paper, we introduce an effective approach to provide an analytical approximation. This approach is validated extensively by comparing it against simulation results under different configurations. It is shown that both the mean delays and the cell loss probabilities obtained from this analysis provide highly accurate estimates.
\end{abstract}


A Refined Approximation of a Finite Capacity Polling System ... Jou, Nilsson, and Lai. 1

\section{Introduction}

The emerging needs for high speed communications and the promise of the technologies to support these services in an integrated fashion have generated a lot of interest in research, development, and standardization of broadband integrated networks. Among the transport and switching techniques for B-ISDN, the Asynchronous Transfer Mode (ATM) technique has shown to be the most promising solution. ATM is a packet oriented transfer mode based on statistical multiplexing in which the information is transported in short, fixed length blocks, referred to as cells, composed of a header and an information field. ATM provides the means for transporting different types of highly bursty traffic such as voice, video images and bulk files. The bandwidth flexibility, the capability to handle all services in a uniform way, and the possible use of statistical multiplexing are advantageous features of ATM. Furthermore, ATM enables a better utilization of the network given the existence of bursty sources.

In this paper, we consider the mean delay and cell loss probability that bursty arrivals incur in an ATM switch architecture as shown in figure 1 [1]. This ATM switch architecture is constructed by connecting self-routing switching modules (SRMs) in a three-stage link configuration which is called a multi-stage self-routing network (MSRN). Each stage of MSRN consists of eight self-routing switching modules. Each module is an $8 \times 8$ crossbar switch which has a finite buffer associated with each crosspoint. The cells in each buffer are transmitted in a cyclic order.

To study the performance of this SRM, we model it by a polling system with cyclic service. In the literature, multiqueue systems served by a single server have been the subject of numerous investigations (see [2], [3] and references therein). Various polling strategies like cyclic or priority service and different types of service disciplines, e.g. exhaustive, gated, or limited service, have been considered. In most of these investigations, the input processes are assumed to be Poisson, and the queues of the polling system are assumed to have infinite capacity. In order to include more realistic modeling elements in the class of polling systems, we consider bursty arrival processes as inputs, and finite buffer capacity in the polling system. 


\section{Multi-Stage Self-Routing Network(MSRN)}

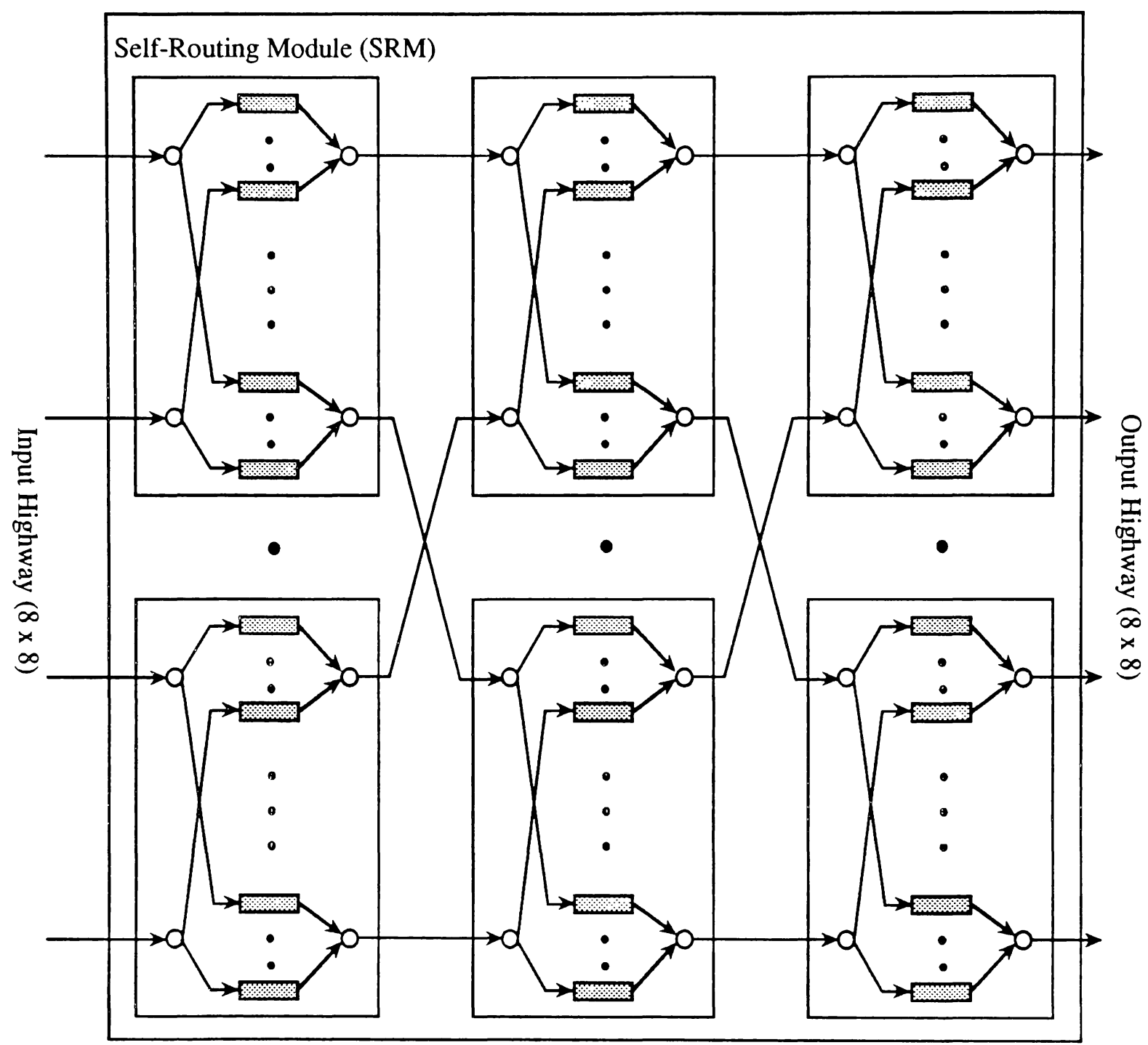

Figure 1: ATM Switching Architecture.

In this paper, we present a queueing model to compute the mean delay and cell loss probability that cells incur in the finite capacity polling system. This model will assume symmetric traffic load, zero switchover time, and 'limited - 1' service [4]. In Section 2 we describe in detail the model we propose. This model will require analysis of the queue length distribution of the polling system and a multiple urn model with uniform occupancy 
A Refined Approximation of a Finite Capacity Polling System … Jou, Nilsson, and Lai. 3 probability which are presented in Sections 3 and 4, respectively. In Section 4, we assume that each queue (urn) can accommodate any number of cells up to its capacity with equal probability. The cell loss probability obtained from this approach overestimates and the mean delay underestimates the simulation result. To have better accuracy, we use the queue length distribution of a single queue in the polling system as the probability for a particular queue to accommodate a certain number of cells. By assuming the independence among the queues, we then take this probability distribution as a basis to compute the occupancy probability of all the possible configurations. In Section 5 , we solve an $I B P / D / 1 / L$ with vacation queueing model and present an algorithm orginated from the closed queueing model to compute the normalization constant. By incorporating the results from these two subsections into the setup of Sections 3 and 4, we are able to provide highly accurate performance measures. Extensive numerical results validated by computer simulations are given in Section 6. Finally, Section 7 presents our conclusions.

\section{Model description}

In this section we describe in detail the switch architecture, the arrival process, and the queueing models which we propose.

\subsection{Switch architecture}

Figure 1 shows the configuration of the MSRN. The MSRN is constructed by connecting SRMs in a three-stage link configuration. In this configuration, there are multiple paths between a first-stage SRM and a third- stage SRM. This configuration allows the traffic flow to be routed efficiently between the input highway and the output highway, and reduces the delay in the switching network. Also, this configuration is inherently reliable because a faulty second stage SRM can be bypassed. Each stage of MSRN consists of eight selfrouting switching modules. Each module is an $8 \times 8$ crossbar switch which has a finite buffer associated with each crosspoint. The SRMs consist of a cell distributor at each inlet, 


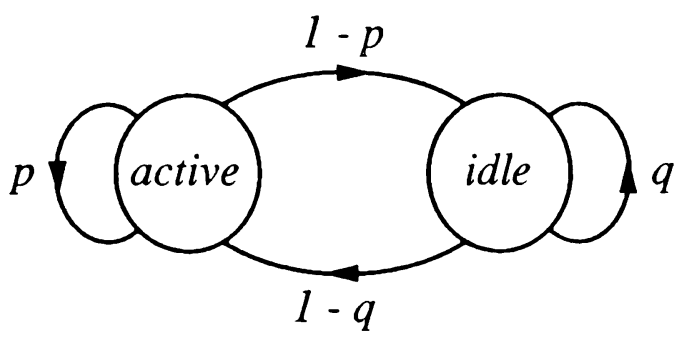

$$
\begin{aligned}
\pi_{A} & =\frac{1-q}{2-p-q} \\
\pi_{I} & =\frac{1-p}{2-p-q}
\end{aligned}
$$

Figure 2: The Markov chain for an IBP

and FIFO buffers for temporarily storing cells in order to resolve outlet contention at each outlet. Cells are assigned to paths (links between SRMs) so that each link carries an equal amount of traffic.

\subsection{Arrival process}

Since most of the traffic sources that an ATM network supports are bursty, a Poisson process may no longer be suitable for describing the network traffic. For instance, interactive data and compressed video generate cells at a near-peak rate for a very short period of time. Immediately, following a near peak rate such a source may become inactive, thus generating no cells. With this scenario, the usual approximation of arrival process by a Poisson process will fail to capture the bursty nature of input traffic and may result in a quite dramatic error in the performance estimation. Kuehn[5] has shown that the system behavior is much more sensitive to arrival processes than to service process. Therefore, we propose to use the IBP which is the generalization of the Interrupted Poisson Process in a discrete time system.

A simple IBP is governed by a Markov chain with two states, an active state and an idle state. The duration of stay in these two states are geometrically distributed. Arrivals occur in a Bernoulli fashion with parameter $\alpha$ when the process is in the active state. No arrivals occur if the process is in the idle state. Given that the process is in the active state (or idle state) at slot $i$, it will remain in the same state in the next slot $i+1$ with probability $p$ (or $q$ ), or will change to the idle state (or active state) with probability $1-p$ (or $1-q$ ). The transitions between the active and idle states are shown in figure 2 , where $\pi_{A}$ and $\pi_{I}$ are the 
A Refined Approximation of a Finite Capacity Polling System ... Jou, Nilsson, and Lai. 5 probabilities that the Markov chain is in the active and idle states, respectively. During the active state, a slot contains a cell with probability $\alpha$. Here we assume $\alpha$ equals 1 which will generate the most bursty traffic.

Letting $t$ be the interarrival time of a cell, it can be shown [6] that the z-transform of the probability distribution of the interarrival time $A(z)=E\left\{z^{t}\right\}$ is

$$
A(z)=\frac{z \alpha[p+z(1-p-q)]}{(1-\alpha)(p+q-1) z^{2}-[q+p(1-\alpha)] z+1} .
$$

The mean interarrival time $E\{t\}$ and the squared coefficient of variation of the time between successive arrivals, $C^{2}$ are as follows:

$$
\begin{aligned}
E\{t\} & =\frac{2-p-q}{\alpha(1-q)} \\
C^{2} & =\frac{\operatorname{Var}(t)}{E\{t\}^{2}} \\
& =1+\alpha\left[\frac{(1-p)(p+q)}{(2-p-q)^{2}}-1\right] .
\end{aligned}
$$

The average arrival rate, i.e. the probability that a slot contains a cell, $\lambda$ is

$$
\lambda=\frac{\alpha(1-q)}{2-p-q} .
$$

By varying $p$ and $q$, we can have different traffic loads and at the same time change the burstiness of the arrival process.

\subsection{Queueing models}

Based on the structure of SRM, we evaluate its performance by using a multiqueue system as shown in figure 3. Under the assumption of symmetric traffic, the arrival processes to the multiqueue system will be characterized also as IBPs with the same parameters of $p$ and $q$ as in the original arrival processes. However, since every original arrival process branches to eight possible outlets, the parameter $\alpha$ of the arrival processes to the polling system will only be one eighth. 


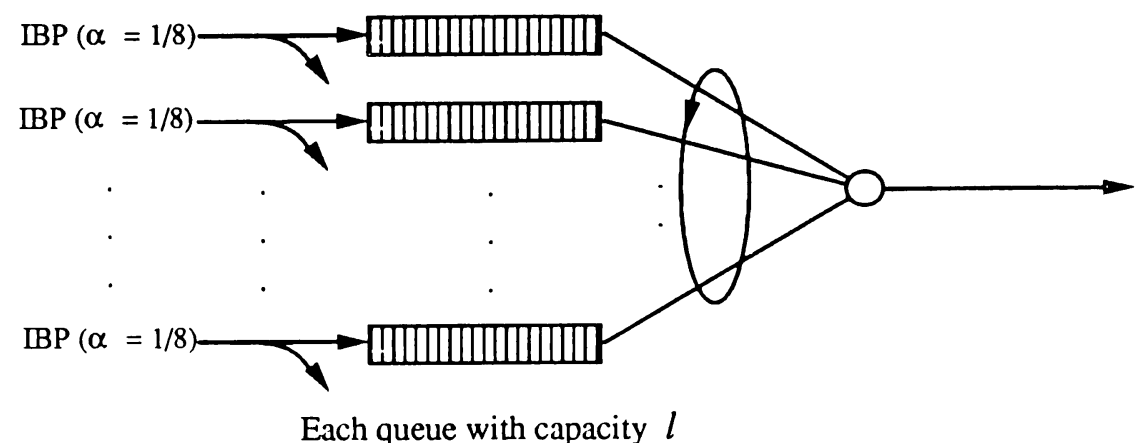

Figure 3: Multiqueue System Served by a Single Server.

Instead of considering the queue lengths of the multiqueue system individually, we will call the distribution of the total number of cells in the polling system as the aggregate queue length distribution. In order to obtain this aggregate queue length distribution, it is necessary to consider the blocking effect due to finite buffer space.

We model the queues in the multiqueue system as multiple urns which have the same limited capacity. Given the number of cells waiting in the system, it is assumed that the occupancy of each queue is independent from each other and the cells are uniformly distributed in any queue, i.e., each position in a queue is equally likely to be occupied. With this model, we can compute the weighting of the cell occupancy configuration which could cause cell loss and then establish the transition matrix which allows us to compute the aggregate queue length distribution. After this distribution is obtained, the mean delay and cell loss probability will follow readily.

Notice that given $R$ cells in the system, the occupancy of these cells in reality will more likely be evenly distributed among these queues because in general the server will visit the queues with longer queue sizes more frequently than the queues with shorter queue sizes. Therefore, given the number of cells in the polling system exceeding a single queue capacity, the occupancy configuration which has at least one full queue is less likely to occur. Hence, the assumption of uniform occupancy will give us a conservative estimate which can serve as an upper bound for cell loss probability of the polling system. 


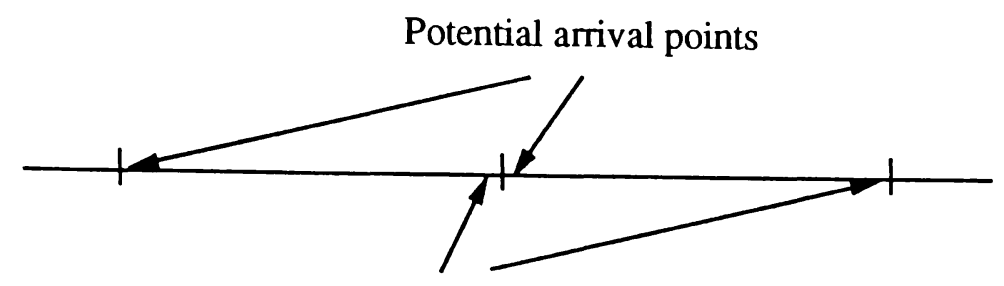

Potential departure points

Figure 4: Potential arrival and departure points.

In order to provide better approximation, we refine the uniform assumption such that the probability of a certain queue position to be occupied is according to the queue length distribution of a single queue. Based upon this refined assumption, we propose a queueing model, $I B P / D / 1 / L$ with vacation, to obtain the queue length distribution of a particular queue in the polling system. This queue length distribution will serve as a basis for computing the weighting of all the possible configurations. The total weighting of all the cell occupancy configurations can be found by a technique originated from the closed queueing model. We use the sum of these weightings as a normalization factor to modify the weighting of the configurations which could cause cell loss. This refined weighting will be incorporated in the steady state equations to compute the aggregate queue length distribution which provides us with highly accurate performance measures.

\section{$3 \quad$ Aggregate queue length distribution}

In our model, we assume that arrivals can only occur at the beginning of each slot and departures leave the system at the end of each slot. This arrangement, as illustrated in figure 4 , is called an early arrival system according to Hunter [8]. During a slot period, one cell may arrive on each input link, and one cell may be transmitted given that the system is not empty. The state change, from active to idle or vice versa, only occurs at the slot point.

Next, the cell arrival process is analyzed for the cells arriving from all inputs in a slot time. We assume that there are $N$ queues in the multiqueue system. The number of active input 
A Refined Approximation of a Finite Capacity Polling System ... Jou, Nilsson, and Lai. 8 links $K$ and arrival cells $M$ in a unit time can both vary from zero to $N$. The probability that the number of active input links is $K$ is

$$
P_{K}(k)=\left(\begin{array}{c}
N \\
k
\end{array}\right) \pi_{A}^{k} \pi_{I}^{N-k}
$$

where $\pi_{A}$ and $\pi_{I}$ denote the probability that an input link is in an active or idle state.

If the number of active input links is $k$, then $m$ cells will arrive in a unit time with probability

$$
P_{M \mid K}(m \mid k)=\left(\begin{array}{c}
k \\
m
\end{array}\right) \alpha^{m}(1-\alpha)^{k-m}
$$

The state transition probability of having $k^{\prime}$ active lines in a slot given $k$ active lines in the previous slot is given by

$$
P_{K^{\prime} \mid K}\left(k^{\prime} \mid k\right)=\sum_{j=0}^{k}\left(\begin{array}{c}
k \\
j
\end{array}\right) p^{j}(1-p)^{k-j}\left(\begin{array}{c}
N-k \\
k^{\prime}-j
\end{array}\right) q^{N-k-k^{\prime}+j}(1-q)^{k^{\prime}-j}
$$

In order to describe the blocking effect, we need to define a conditional probability $P\left(m^{\prime \prime} \mid m^{\prime}, q s i z e\right)$ as

$P\left\{m "\right.$ cells accepted $\mid m^{\prime}$ cells arrived and qsize cells in the system before arrivals $\}$.

This probability will be further described and computed by a multiple urn model which is discussed in the next section.

Now, we define a two dimensional state variable $(K, Q)$ such that the queue length becomes $Q$ as the result of having $M^{\prime}$ cells arrive and $M^{\prime \prime}$ cells accepted in a slot given that $K$ input lines are active. The state probability $P_{K, Q}(k, q s i z e)$ can be obtained by a numerical solution of the following steady state equations:

$$
P_{K, Q}\left(k^{\prime}, q s i z e^{\prime}\right)=\sum_{k=0}^{N} \sum_{m^{\prime}=0}^{k^{\prime}} \sum_{q s i z e=0}^{Q_{m}} P_{K, Q}(k, q s i z e) P_{K^{\prime} \mid K}\left(k^{\prime} \mid k\right) P_{M \mid K}\left(m^{\prime} \mid k^{\prime}\right) P\left(m^{\prime \prime} \mid m^{\prime}, q s i z e^{\prime \prime}\right),
$$


A Refined Approximation of a Finite Capacity Polling System … Jou, Nilsson, and Lai. 9

$$
\sum_{k=0}^{N} \sum_{q s i z e=0}^{Q_{m}} P_{K, Q}(k, q s i z e)=1
$$

where $Q_{m}$ is the total capacity of the multiqueue system. In equation (2), qsize" and qsize' are given by $q s i z e "=\max (q s i z e-1,0)$ and $q s i z e^{\prime}=m "+q s i z e "$, respectively. From $P_{K, Q}(k, q s i z e)$, we can sum over $K$ and find the queue length distribution $P_{Q}(q s i z e)$. Since we have assumed zero switchover time in the system, the mean output rate $\lambda_{\text {out }}$ can be determined as

$$
\begin{aligned}
\lambda_{\text {out }} & =1-P_{Q}(0) \\
& =\lambda_{\text {in }}\left(1-P_{\text {loss }}\right) .
\end{aligned}
$$

Therefore, the cell loss probability $P_{\text {loss }}$ is obtained as

$$
P_{\text {loss }}=1-\frac{\lambda_{\text {out }}}{\lambda_{\text {in }}}
$$

After we compute the mean queue length $L$, the mean delay $W$ can be determined by using Little's result as

$$
W=\frac{L}{\lambda_{\text {out }}}
$$

\section{Multiple urn model}

In order to compute the conditional probability $P\left(m^{\prime \prime} \mid m^{\prime}, q s i z e\right)$ defined in the last section, we propose a multiple urn model to find the total number of ways to place $r$ indistinguishable balls into $n$ distinguishable boxes given that the capacity of each box is limited to $l$. It is shown [9] that given $r \leq l$ (i.e. no capacity limit), the number of distinguishable distributions is

$$
A_{n, r}=\left(\begin{array}{c}
n+r-1 \\
r
\end{array}\right)=\left(\begin{array}{c}
n+r-1 \\
n-1
\end{array}\right)
$$


A Refined Approximation of a Finite Capacity Polling System ... Jou, Nilsson, and Lai. 10 Define $B_{n, r, k}$ as the number of ways of having at least $k_{i}$ balls in $i^{\text {th }}$ box (no limit). Then $B_{n, r, k}$ can be easily found as the following

$$
B_{n, r, k}=\left(\begin{array}{c}
n+r-k-1 \\
r-k
\end{array}\right)=\left(\begin{array}{c}
n+r-k-1 \\
n-1
\end{array}\right), \quad k=\sum_{i=1}^{n} k_{i}
$$

From the principle of inclusion and exclusion we can obtain the total number of different configurations in this multiple urn model as

$$
\begin{aligned}
T_{n, r, l} & =\sum_{i=0}^{n}(-1)^{i}\left(\begin{array}{c}
n \\
i
\end{array}\right)\left(\begin{array}{c}
n+r-i(l+1)-1 \\
n-1
\end{array}\right) \\
& =\sum_{i=0}^{n}(-1)^{i}\left(\begin{array}{c}
n \\
i
\end{array}\right) B_{n, r-i(l+1), 0},
\end{aligned}
$$

where $B_{n, r, 0}$ is equal to $A_{n, r}$ which denotes the total number of solutions given no limit imposed on the capacity of boxes. $B_{n, r-(l+1), 0}$ represents the number of configurations in which at least one of the $n$ boxes contains no less than $l+1$ balls. This condition violates the limit and should be subtracted from $B_{n, r, 0}$. The rest of the terms is just to compensate the over subtraction that $B_{n, r-(l+1), 0}$ introduces.

We now proceed to find $C_{n, r, l}$, the number of ways of having at least one full box for this multiple urn model. If the total number of balls $r$ is less than $l, C_{n, r, l}$ equals zero. For $l \leq r<2 l$, it is easy to verify that

$$
C_{n, r, l}=n B_{n-1, r-l, 0}
$$

Likewise, when $2 l \leq r<3 l$

$$
\begin{aligned}
C_{n, r, l} & =\left(\begin{array}{c}
n \\
2
\end{array}\right) B_{n-2, r-2 l, 0}+\left(\begin{array}{c}
n \\
1
\end{array}\right)\left[T_{n-1, r-l, l}-\left(\begin{array}{c}
n-1 \\
1
\end{array}\right) B_{n-2, r-2 l, 0}\right] \\
& =\left(\begin{array}{c}
n \\
2
\end{array}\right) T_{n-2, r-2 l, l}+\left(\begin{array}{c}
n \\
1
\end{array}\right)\left(T_{n-1, r-l, l}-C_{n-1, r-l, l}\right),
\end{aligned}
$$

where the first term on the right hand side of equation (3) denotes the number of ways to have two full boxes, and the second term gives the number of ways of having one full box. 
A Refined Approximation of a Finite Capacity Polling System ... Jou, Nilsson, and Lai. 11 Proceeding in the same manner, we conclude that for $k l \leq r<(k+1) l$

$$
C_{n, r, l}=\sum_{i=1}^{k}\left(\begin{array}{c}
n \\
i
\end{array}\right)\left(T_{n-i, r-i * l, l}-C_{n-i, r-i * l, l}\right),
$$

where $C_{n, r, l}$ is obtained recursively. Notice that the expression in the summation in equation (4) denotes the number of ways to have exactly $i$ full boxes.

According to the definition of $P\left(m^{\prime \prime} \mid m^{\prime}, q s i z e\right)$, we now obtain the conditional probability as

$$
P\left(m^{\prime \prime} \mid m^{\prime}, r\right)=\frac{\sum_{i=0}^{\lfloor\lceil\rfloor} \frac{\left(\begin{array}{c}
N-i \\
m "
\end{array}\right)\left(\begin{array}{c}
i \\
m^{\prime}-m^{\prime}
\end{array}\right)}{\left(\begin{array}{c}
N \\
m^{\prime}
\end{array}\right)}\left(\begin{array}{c}
N \\
i
\end{array}\right)\left(T_{N-i, r-i \neq l, l}-C_{N-i, r-i * l, l}\right)}{T_{N, r, l}} .
$$

\section{Refinement of the Approximation}

From the computations in Sections 3 and 4, we find out that the cell loss probabilities obtained from the analytical model overestimate the results obtained from simulation. On the other hand, the mean delays tend to underestimate. These phenomena are due to the assumption of equal probability among the configurations of occupancy in the multiple urn model. Based upon this observation, we solve an $I B P / D / 1 / L$ with vacation queueing model and formulate the derivation to compute the normalization factor in order to refine the approximation as we discussed in Section 2.3.

\section{1 $I B P / D / 1 / L$ with Vacation}

If we focus on a specific queue in the polling system, the queueing model of this particular queue can be identified as an $I B P / D / 1 / L$ with vacation where $L$ denotes the capacity of this queue. To solve this queueing model, we need the aggregate queue length distribution of the polling system in order to characterize the vacation time distribution of this single queue. The aggregate queue length distribution of the polling system was obtained by using 
A Refined Approximation of a Finite Capacity Polling System ... Jou, Nilsson, and Lai. 12 the algorithm implemented in Sections 3 and 4. The vacation time, or to be more precise, the cycle time distribution defined by $c(k)$ which refers to the time between two immediate visits of the server can be computed by the equations in Section 4 as follows.

Define $D_{i, j}$ as the number of configurations which have $i$ empty queues given that there are $j$ cells in the system. Or equivalently, we can define $D_{i, j}$ as the number of configurations which have $i$ queues full of holes given that there are $N \times L-j$ holes in the system. Therefore, $D_{i, j}$ can be expressed as

$$
D_{i, j}=\left(\begin{array}{c}
N \\
i
\end{array}\right)\left(T_{N-i,(N \times L-j)-i * L, L}-C_{N-i,(N \times L-j)-i * L, L}\right)
$$

The cycle time distribution, $c(k)$, can be obtained as

$$
c(k)=\sum_{j=1}^{N \times L} \frac{D_{N-k, j} P_{Q}(j)}{T_{N, j, L}}+1_{\{k=1\}} P_{Q}(0)
$$

where $1 \leq k \leq N$.

We now proceed to solve the queueing model of $I B P / D / 1 / L$ with vacation. The basic techniques used in this derivation are similar to those used in [10]. The steady state distribution of queue length is computed by the embedded Markov chain approach. Cells arrive at the system in accordance with an IBP with parameters $\alpha, p$, and $q$. There is a single server whose service time is deterministic of one slot. If an arriving cell finds exactly $L$ cells in the system, then this cell is blocked (lost) without being served.

The system will be examined at time epochs $\left\{t_{0}, t_{1}, \cdots\right\}$ of service completion or vacation termination. The state space of the system is $\left\{R_{i}, \xi_{i}, \eta_{i}\right\}$, defined as follows. $R_{i}$ is the number of cells in the system at the embedded point $t_{i}$. If the point $t_{i}$ is a vacation termination instant, i.e., the point when the server starts to scan this particular queue, then $\xi_{i}=0$; otherwise $\xi_{i}=1$. The state of the arrival process at the embedded point $t_{i}$ is denoted by $\eta_{i}$, where $\eta_{i}$ is 1 if the IBP is in its active state or 0 if it is in the idle state. The state transitions occur at cell departure instants or vacation termination instants. Let $X_{r, \eta}$ and $Y_{r, \eta}$ represent 
A Refined Approximation of a Finite Capacity Polling System ... Jou, Nilsson, and Lai. 13 the limiting probability distributions defined as follows

$$
\begin{array}{rlrl}
X_{r, \eta} & \equiv \lim _{i \rightarrow \infty} P_{r}\left\{R_{i}=r, \xi_{i}=1, \eta_{i}\right\} & & 0 \leq r \leq L-1, \\
Y_{r, \eta} \equiv \lim _{i \rightarrow \infty} P_{r}\left\{R_{i}=r, \xi_{i}=0, \eta_{i}\right\} & 0 \leq r \leq L .
\end{array}
$$

It is easy to verify that $X_{r, \eta}=Y_{r+1, \eta}, 0 \leq r \leq L-1$. Therefore, we can set up the steady state equations by focusing only on $Y_{r, \eta}$.

Define $g(j, n, k \mid i)$ as the probability of having $n$ active slots among $k$ transition slots with the arrival process starting at state $i$ and ending at state $j$ between two immediate vacation termination instants. This probability can be obtained recursively by the following equations:

$$
\begin{aligned}
& g(0, n, k \mid 0)=q g(0, n, k-1 \mid 0)+(1-p) g(1, n, k-1 \mid 0), \\
& g(1, n, k \mid 0)=(1-q) g(0, n-1, k-1 \mid 0)+p g(1, n-1, k-1 \mid 0), \\
& g(0, n, k \mid 1)=q g(0, n, k-1 \mid 1)+(1-p) g(1, n, k-1 \mid 1), \\
& g(1, n, k \mid 1)=(1-q) g(0, n-1, k-1 \mid 1)+p g(1, n-1, k-1 \mid 1),
\end{aligned}
$$

where the initial conditions are given by

$$
\begin{array}{ll}
g(0,0,1 \mid 0)=q, & g(0,1,1 \mid 0)=0, \\
g(1,0,1 \mid 0)=0, & g(1,1,1 \mid 0)=1-q, \\
g(0,0,1 \mid 1)=1-p, & g(0,1,1 \mid 1)=0, \\
g(1,0,1 \mid 1)=0, & g(1,1,1 \mid 1)=p .
\end{array}
$$

We further define $G(j, r, k \mid i)$ as the probability of having $r$ cells arriving in $k$ transition slots with the arrival process starting at state $i$ and ending at state $j$ between two immediate vacation termination instants. The probability $G(j, r, k \mid i)$ can be expressed in terms of $g(j, n, k \mid i)$ as

$$
G(j, r, k \mid i)=\sum_{n=0}^{N}\left(\begin{array}{l}
n \\
r
\end{array}\right) \alpha^{r}(1-\alpha)^{n-r} g(j, n, k \mid i)
$$


A Refined Approximation of a Finite Capacity Polling System ... Jou, Nilsson, and Lai. 14 When the system is under steady state, the limiting probability distribution $Y_{r, \eta}$ satisfies the following equations:

$$
\begin{aligned}
& Y_{r, 0}=Y_{0,0} H(0, r \mid 0)+Y_{0,1} H(0, r \mid 1)+\sum_{k=0}^{r}\left(Y_{k+1,0} H(0, r-k \mid 0)+Y_{k+1,1} H(0, r-k \mid 1)\right), \\
& Y_{r, 1}=Y_{0,0} H(1, r \mid 0)+Y_{0,1} H(1, r \mid 1)+\sum_{k=0}^{r}\left(Y_{k+1,0} H(1, r-k \mid 0)+Y_{k+1,1} H(1, r-k \mid 1)\right),
\end{aligned}
$$

where the transition probability $H(j, r \mid i)$ is given by

$$
H(j, r \mid i)=\sum_{k=1}^{N} G(j, r, k \mid i) c(k),
$$

which represents the probability of having $r$ arrivals between two vacation termination instants. The arrival process at these two embedded points is in the state $i$, and $j$, respectively. Combining the steady state equations (5) and (6) together with the normalization equation

$$
\sum_{r=0}^{L} \sum_{\eta=0}^{1}\left(X_{r, \eta}+Y_{r, \eta}\right)=1
$$

we can easily solve $X_{r, \eta}$ and $Y_{r, \eta}$. (For simplity, we assume $X_{L, \eta}=0$.) The queue length distribution $P_{I B P_{-} Q}$ is followed readily as

$$
P_{I B P_{-} Q}(r)=\sum_{\eta=0}^{1}\left(X_{r, \eta}+Y_{r, \eta}\right), \quad 0 \leq r \leq L .
$$

\subsection{Computing the Normalization Factor}

We recognize that the discrepancy of the results obtained from Sections 3 and 4 is due to the assumption that each position in a queue has the same probability to be occupied. In other words, the queue length distribution under this assumption is $1 / L$ uniformly. This is of course not the case in reality. In order to refine this assumption, we use the queue length distribution $P_{I B P_{-} Q}(r)$ found in Section 5.1 as the probability for a particular queue 
A Refined Approximation of a Finite Capacity Polling System ... Jou, Nilsson, and Lai. 15 to accommodate $r$ cells. Given $R$ cells in the polling system, the stationary distribution of the system state $\underline{\mathbf{r}}=\left(r_{1}, r_{2}, \cdots, r_{N}\right)$ is equal to

$$
\begin{aligned}
P(\underline{\mathbf{r}}) & =P\left(r_{1}, r_{2}, \cdots, r_{N}\right) & & \\
& =\frac{1}{G} \prod_{i=1}^{N} P_{i}\left(r_{i}\right), & & \sum_{i=1}^{N} r_{i}=R
\end{aligned}
$$

where the states of the various queues are assumed to be independent. $P_{i}\left(r_{i}\right)$ in equation (7) is given by $P_{I B_{-} Q}(r)$. This setup is exactly the same as the case in the closed queueing network except that we have finite capacity in each queue, i.e. $r_{i} \leq L$, rather than $r_{i} \leq R$ in the general cases. Following the same spirit, we extend the derivation given in [11] to compute the normalization factor $G_{N}(R)$ recursively. In particular, we have

$$
\begin{array}{rll}
G_{1}(R) & =P_{1}(R) & 0 \leq R \leq L, \\
G_{2}(R) & =\sum_{r_{2}=\max (0, R-L)}^{\min (L, R)} P_{2}\left(r_{2}\right) G_{1}\left(R-r_{2}\right) & \\
& \vdots & \\
G_{N}(R) & =\sum_{r_{N}=\max [0, R-(N-1) \times L]}^{\min (L, R)} P_{N}\left(r_{N}\right) G_{N-1}\left(R-r_{N}\right) & 0 \leq R \leq N \times L .
\end{array}
$$

This recurrence relation allows $G_{N}(R)$ to be computed in $O\left(N R^{2}\right)$ steps.

The above equations are subject to the constraint of having $L$ spaces in each queue. In order to refine the conditional blocking probability in Section 3, we need another set of normalization factor $\overline{G_{N}}(R)$ which follows the same derivation but is subject to the constraint of $L-1$ buffer spaces. When $R>N \times(L-1)$, we simply let $\overline{G_{N}}(R)$ be zero.

Define $P_{\text {full }}(K, R)$ as the probability of having $R$ cells in the polling system and $K$ out of $N$ queues full. Therefore, we have

$$
P_{\text {full }}(0, R)=\frac{\overline{G_{N}}(R)}{G_{N}(R)}
$$


A Refined Approximation of a Finite Capacity Polling System ... Jou, Nilsson, and Lai. 16

$$
P_{\text {full }}(K, R)=\frac{\left(\begin{array}{c}
N \\
K
\end{array}\right) G_{K}(K \times L) \overline{G_{N-K}}(R-K \times L)}{G_{N}(R)}, \quad 1 \leq K \leq N .
$$

The refined conditional blocking probability $P\left(m^{\prime \prime} \mid m^{\prime}, R\right)$ can be expressed as

$$
P\left(m^{\prime \prime} \mid m^{\prime}, R\right)=\sum_{K=0}^{\left\lfloor\frac{R}{L}\right\rfloor} \frac{\left(\begin{array}{c}
K \\
m^{\prime}-m^{\prime \prime}
\end{array}\right)\left(\begin{array}{c}
N-K \\
m^{\prime \prime}
\end{array}\right) P_{f u l l}(K, R)}{\left(\begin{array}{c}
N \\
m^{\prime}
\end{array}\right)}
$$

This blocking probability can be inserted into equation (2) in Section 3 to obtain the desired performance measures.

\section{Numerical results}

In this section, we examine several configurations where the presented approximations are compared against the simulation results. The performance measures, mean delay, and cell loss probability are affected by the buffer capacity of the multiqueue system and the burstiness of the arrival processes. In terms of queue capacity, we present three cases where the buffer sizes are 4,8 , and 16 , respectively.

In order to show the effect of the arrival burstiness, we vary the squared coefficient of variation $\left(C^{2}\right)$ of the arrival processes from 1 , to 20 , to 200 . These three kinds of burstiness represent three typical cases. When $C^{2}$ equals 1 , we can regard this arrival process as being smooth. The burstiness of voice is represented by the case where $C^{2}$ equals 20 . We use $C^{2}=200$ for the burstiness of data traffic.

Figures 5, 7, and 9 show the mean delay times that the arrivals incur under different burstinesses when the queue capacities are 4,8 , and 16 , respectively. The worst case is where $C^{2}$ equals 200 and the arrival rate is 0.9 . In this worst case, the relative errors between the analytical results and the simulations are $3.2 \%, 5.0 \%$, and $5.1 \%$ when the queue capacities are 4,8 , and 16 , respectively. It is clearly shown that the analysis follows the simulation 
A Refined Approximation of a Finite Capacity Polling System ... Jou, Nilsson, and Lai. 17 closely. Comparing these three figures, we can see that for the larger queue capacity the bursty effect becomes more obvious.

The impact of the burstiness toward the cell loss probability can be seen in figures 6 , 8 , and 10 where the buffer capacities vary from 4 to 16 . From these figures, we see that the analysis traces the simulation nicely when the queue capacities are 4 and 8 . With buffer capacity of 16 , the analytic results could be twice as high as the simulations. From our experiments, the larger the queue capacity is, the higher the discrepancy. However, a benefit of this analysis is that it overestimates the cell loss probability and provides us with a conservative approximation. Notice that the case where the queue capacity is 16 and $C^{2}$ $=1$ is omitted due to the fact that there is no cell loss recorded in the simulation except when the traffic load is 0.9 .

Figures 11 to 16 compare the system performance under different queue capacities when $C^{2}$ is fixed. From this comparison, we may be able to decide the capacity which meets the performance requirements under the ATM environment.

\section{Conclusion}

A realistic polling system with finite capacity does not lend itself to an exact analysis. In this paper, we presented an effective approach to provide an analytical approximation. As for the arrival processes, we also take into account the effect of bursty arrivals which is an essential feature in the ATM environment.

It is shown that the analytical model works very well with a wide range in both the burstiness and the traffic load of the arrival process as well as the queue capacity of the polling system. The analysis is also computationally effective. The major portion of the computation time of this analysis is devoted to solving the steady state equations in order to obtain the aggregate queue length distribution. It is our experience that the speedup of the analysis over simulation on average is more than two orders of magnitude.

The strength of this analysis is that it is able to provide accurate performance measures 
A Refined Approximation of a Finite Capacity Polling System ... Jou, Nilsson, and Lai. 18 in a short period of time. Therefore, it can be applied to decide the system specifications in order to meet the performance requirements under ATM environment without running the simulations. 
A Refined Approximation of a Finite Capacity Polling System ... Jou, Nilsson, and Lai. 19

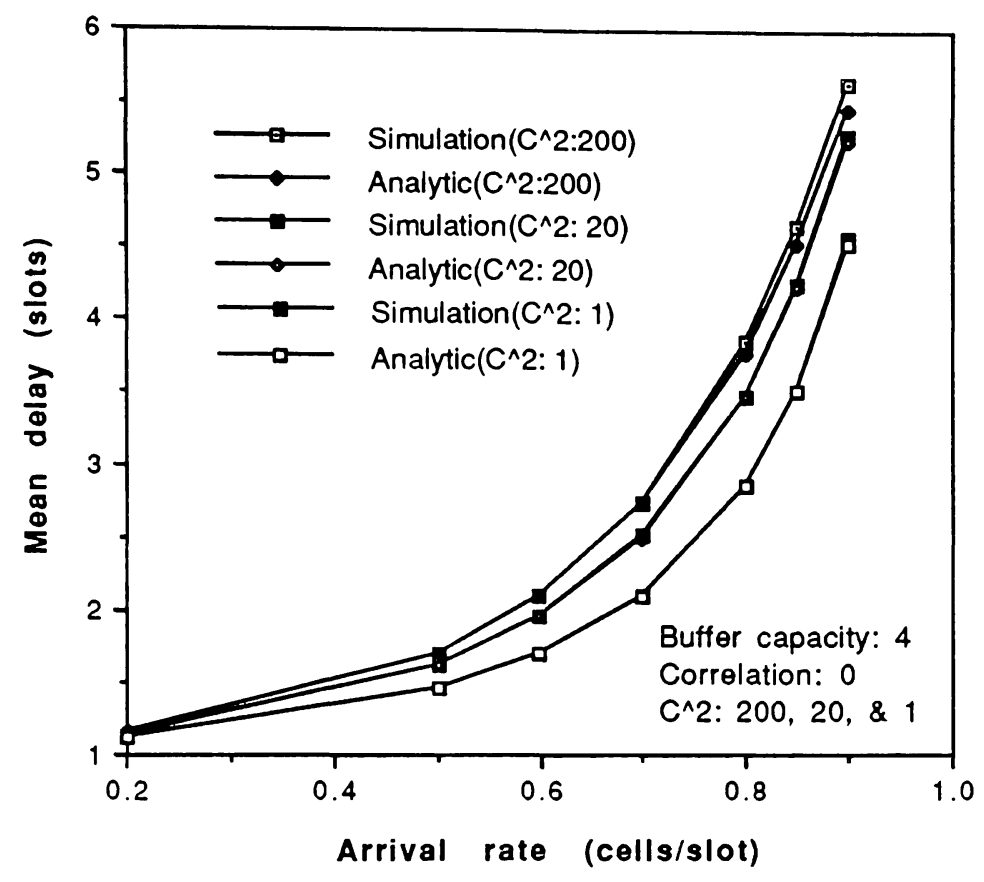

Figure 5: Mean delays incurred in the polling system when queue capacity $=4$.

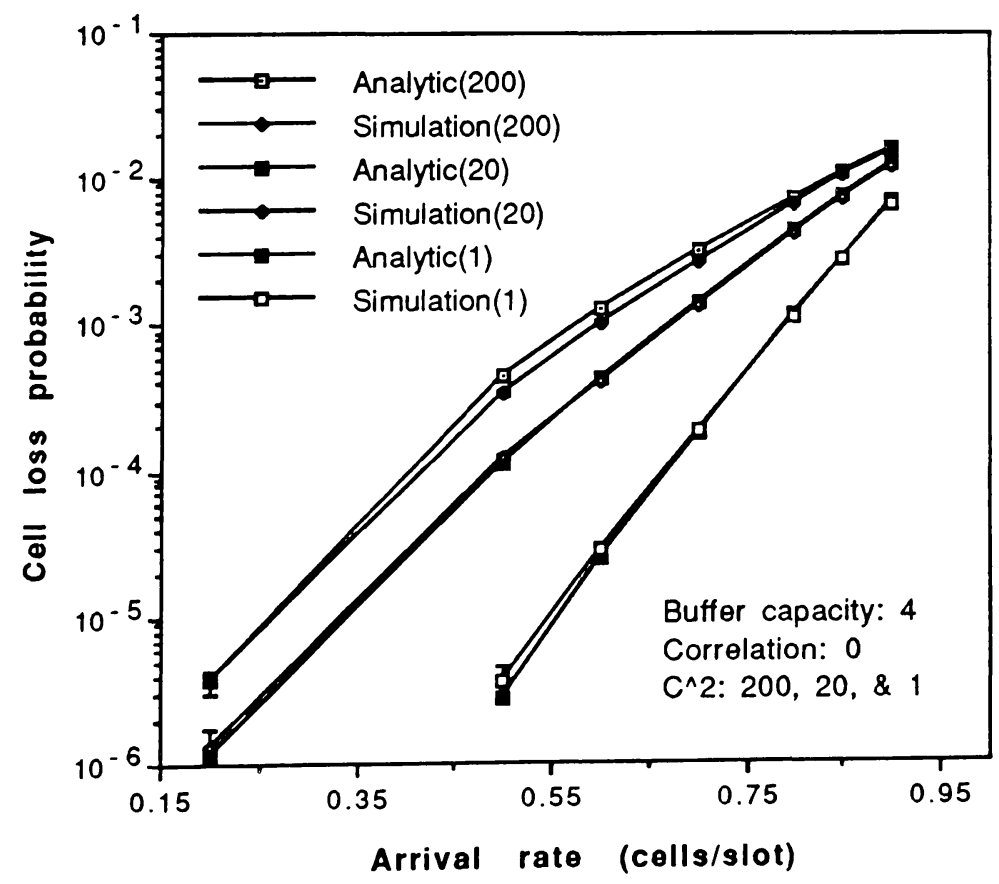

Figure 6: Cell loss probability incurred in the polling system when queue capacity $=4$. 


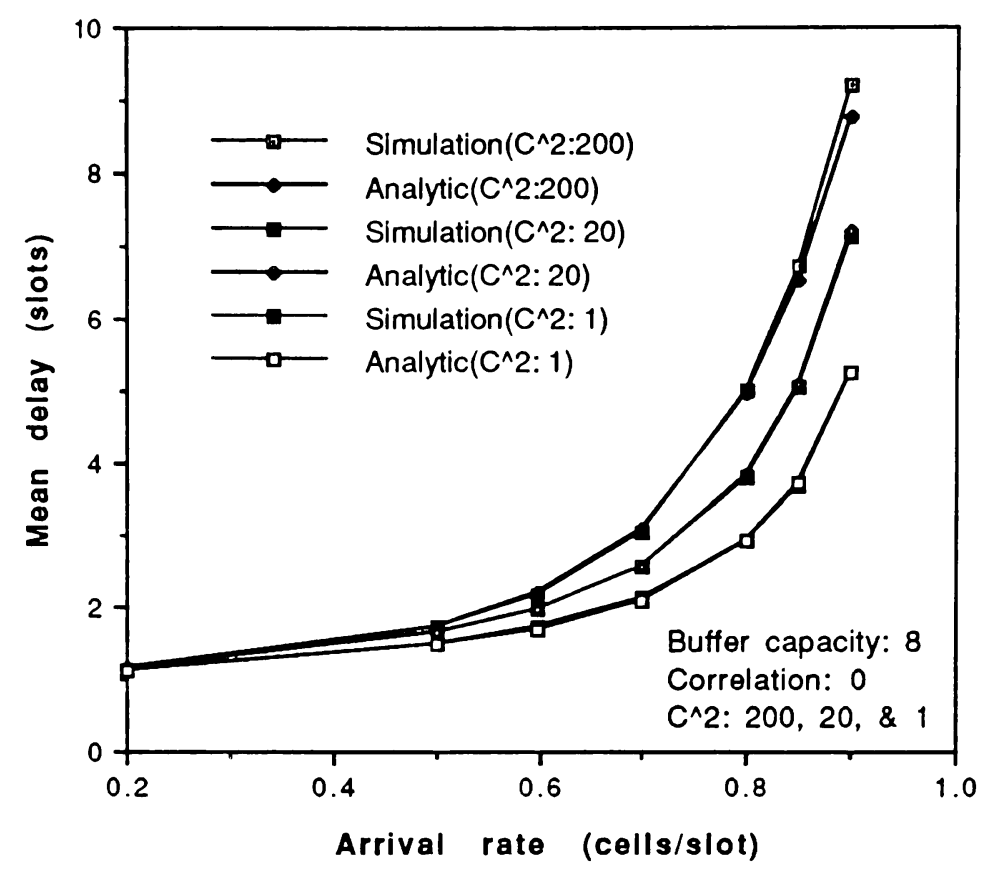

Figure 7: Mean delays incurred in the polling system when capacity $=8$.

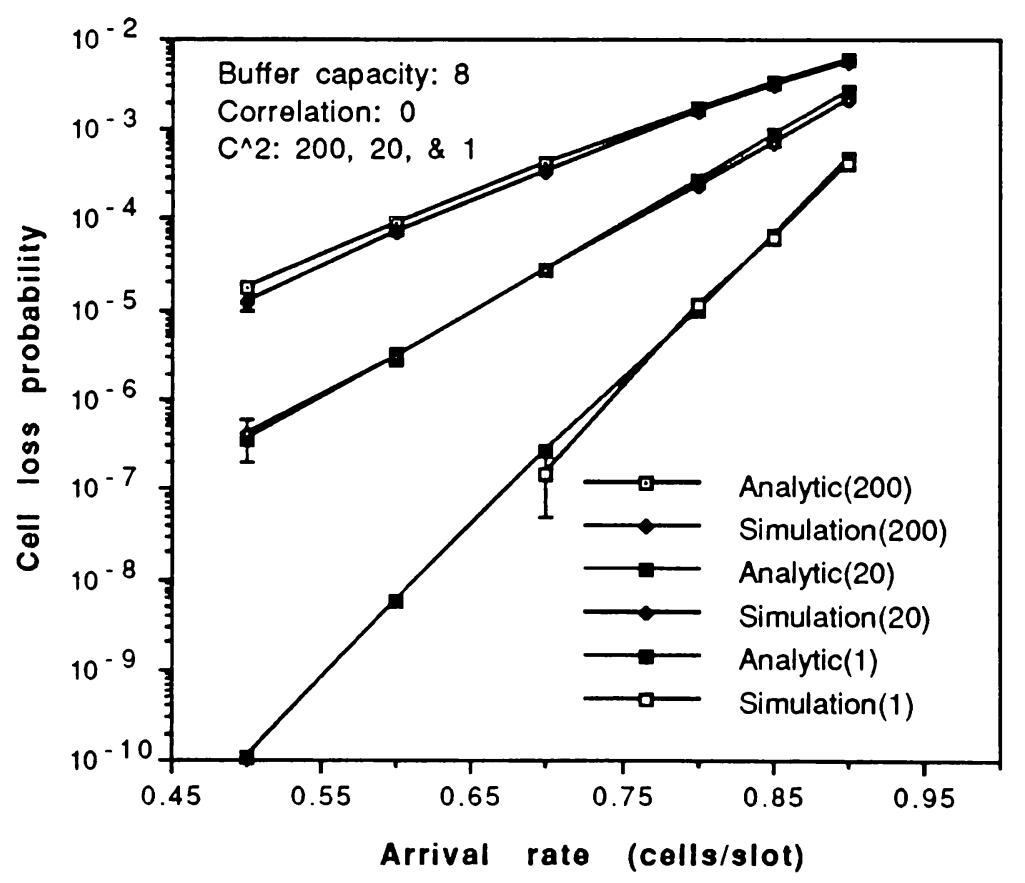

Figure 8: Cell loss probability incurred in the polling system when capacity $=8$. 
A Refined Approximation of a Finite Capacity Polling System ... Jou, Nilsson, and Lai. 21

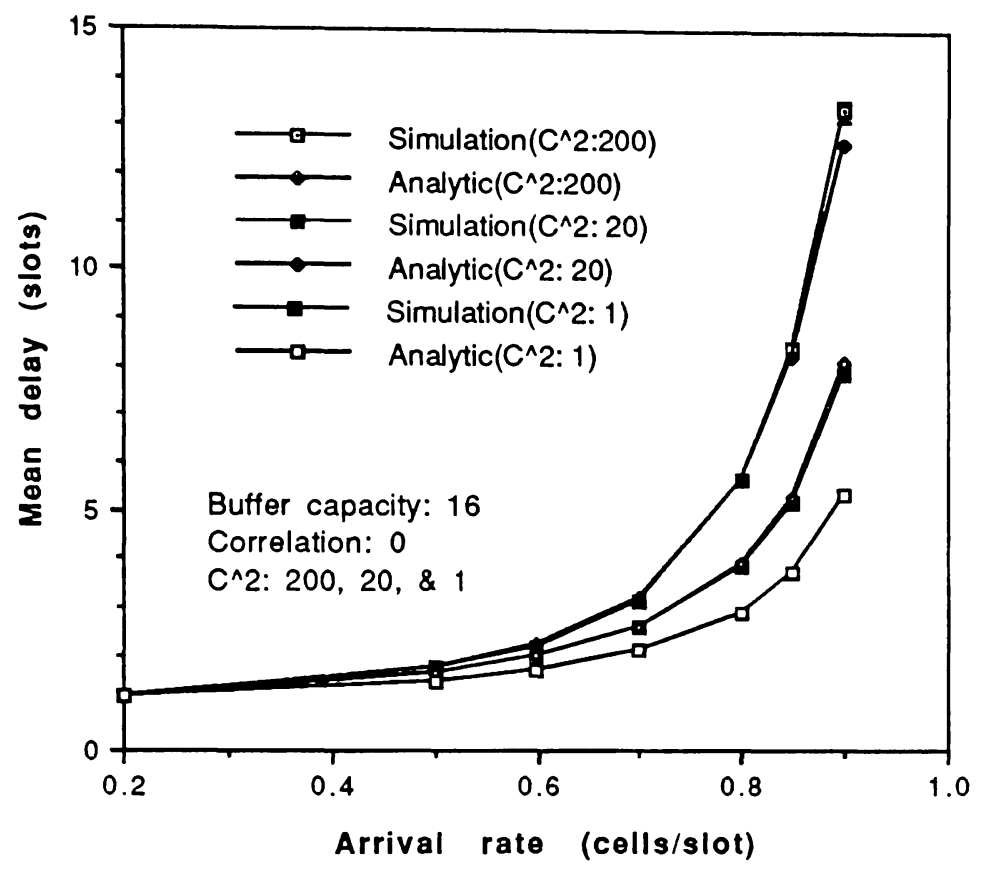

Figure 9: Mean delays incurred in the polling system when queue capacity $=16$.

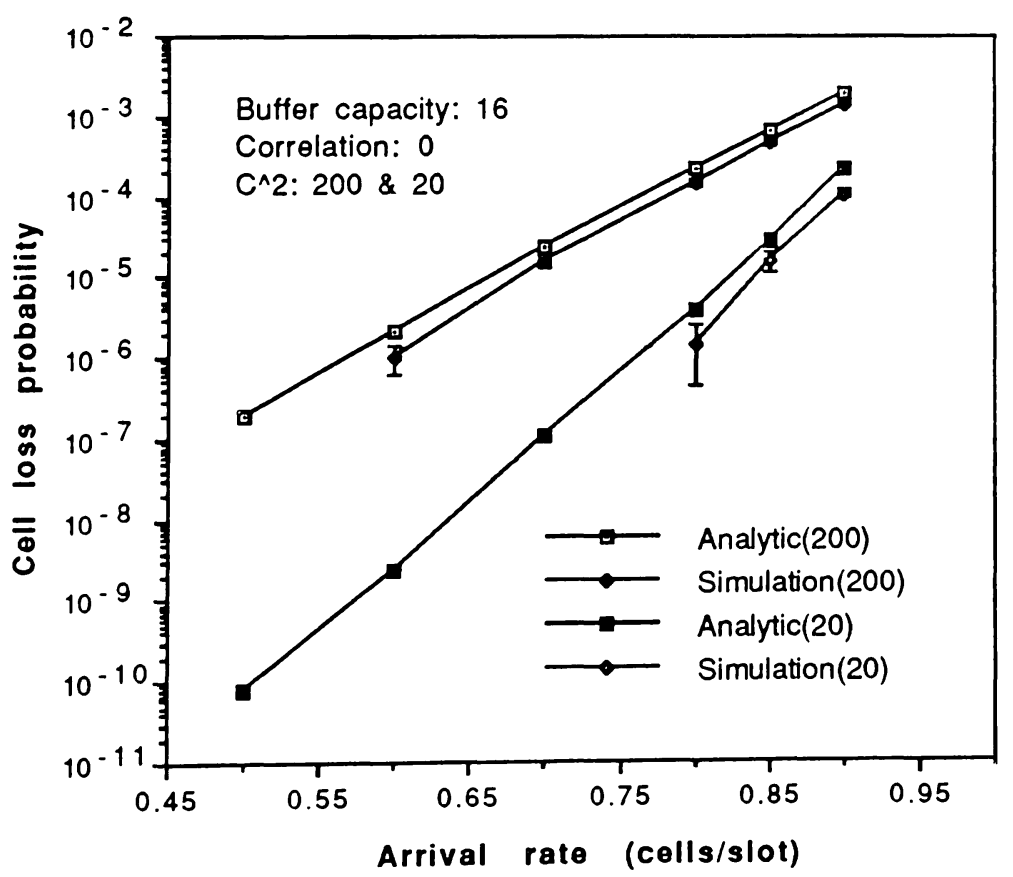

Figure 10: Cell loss probability incurred in the polling system when capacity $=16$. 
A Refined Approximation of a Finite Capacity Polling System ... Jou, Nilsson, and Lai. 22

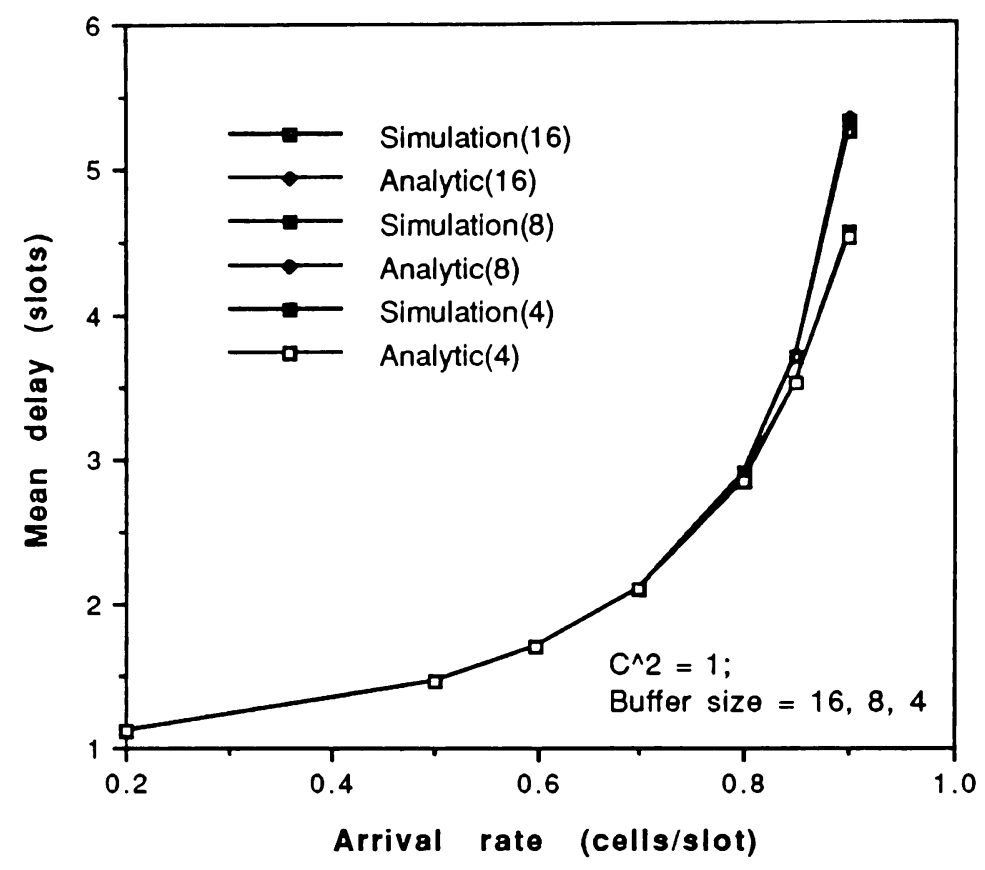

Figure 11: Mean delays incurred in the polling system when $C^{2}=1$.

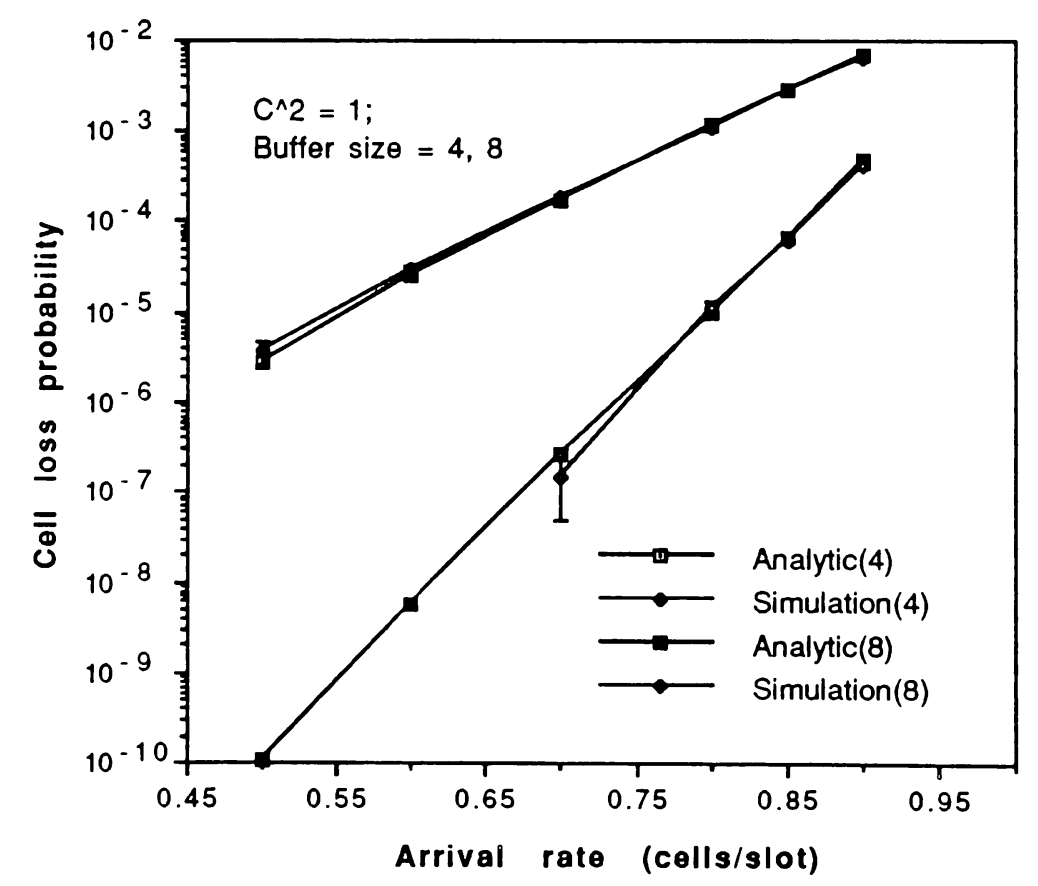

Figure 12: Cell loss probability incurred in the polling system when $C^{2}=1$. 
A Refined Approximation of a Finite Capacity Polling System ... Jou, Nilsson, and Lai. 23

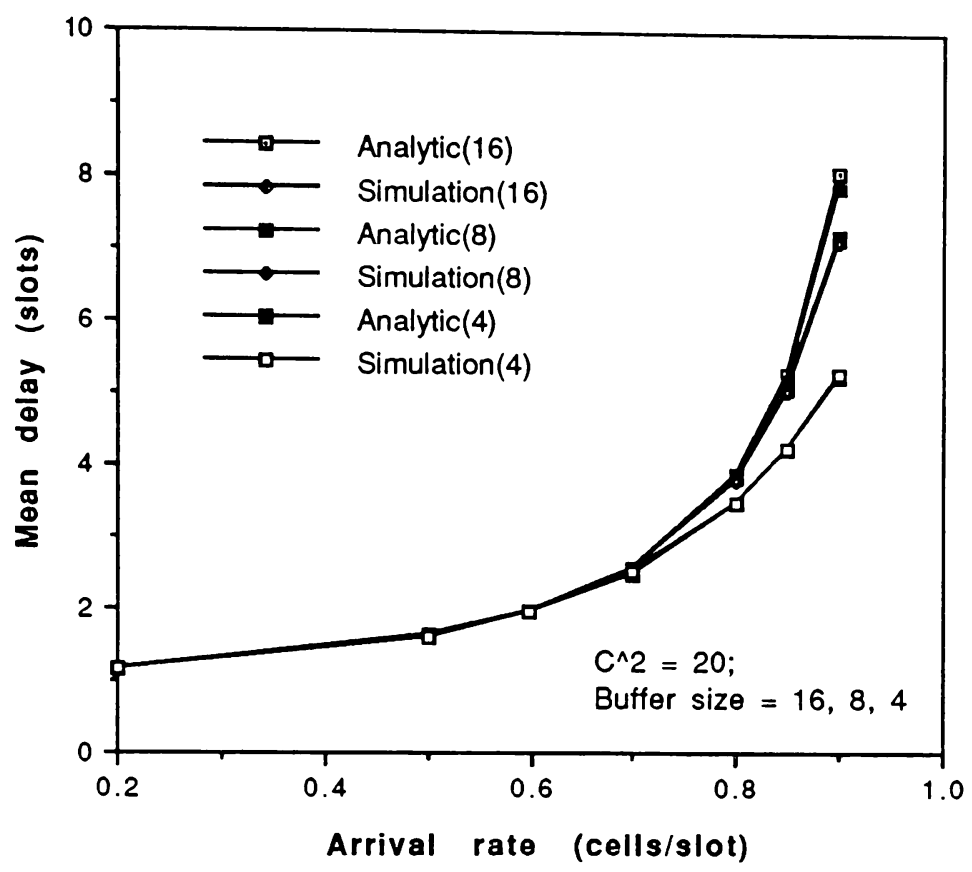

Figure 13: Mean delays incurred in the polling system when $C^{2}=20$.

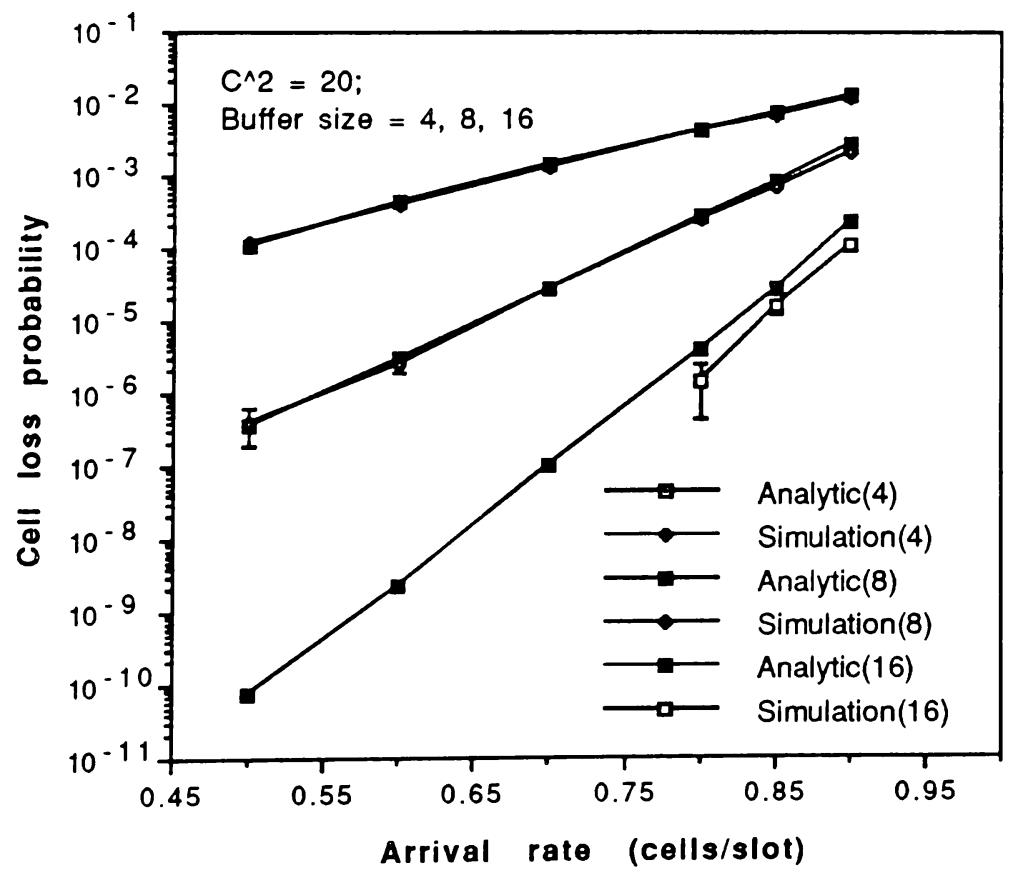

Figure 14: Cell loss probability incurred in the polling system when $C^{2}=20$. 


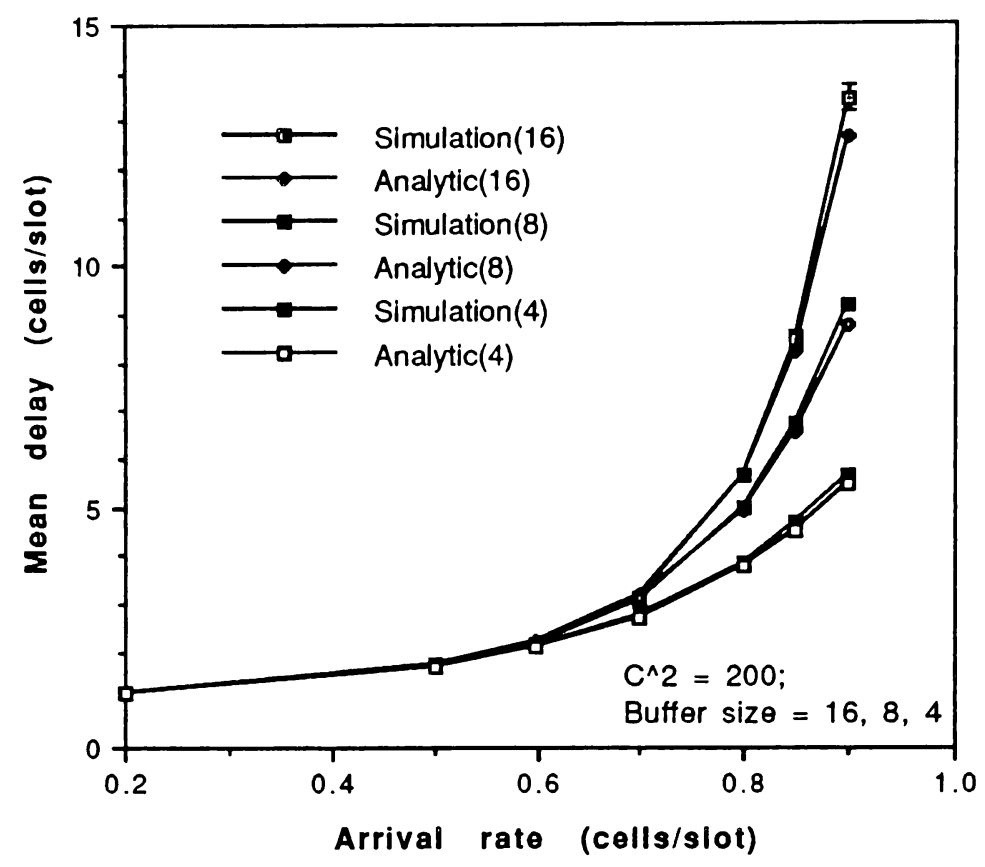

Figure 15: Mean delays incurred in the polling system when $C^{2}=200$.

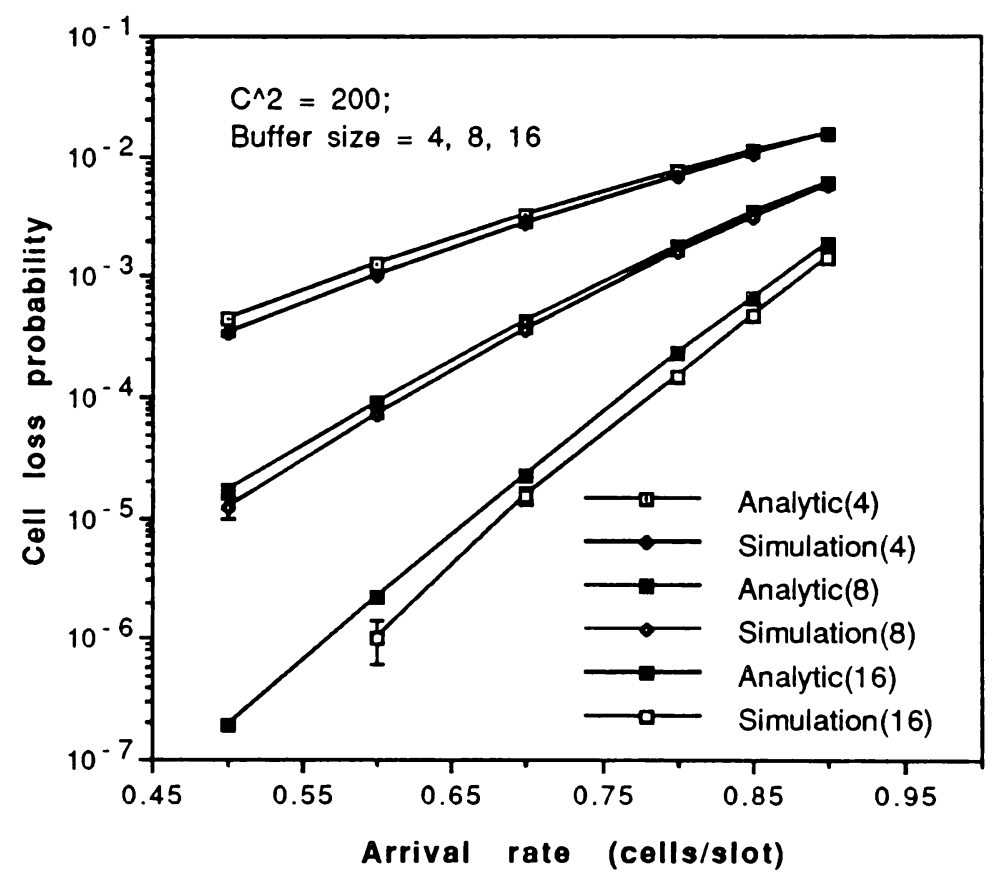

Figure 16: Cell loss probability incurred in the polling system when $C^{2}=200$. 
A Refined Approximation of a Finite Capacity Polling System ... Jou, Nilsson, and Lai. 25

\section{References}

[1] K. Hajikano, K. Murakami, E. Iwabuchi, O. Isono, and T. Kobayashi, "Asynchronous transfer mode switching architecture for broadband ISDN - multistage self-routing switching," IEEE International Conference on Communications, vol. 2, pp. 911-915, 1988.

[2] H. Takagi, "Queueing analysis of polling models: an update," Stochastic Analysis of Computer and Communication Systems, pp. 267-318, H. Takagi (editor), Elsevier Science Publishers B.V. (North-Holland) Amsterdam, 1990.

[3] P. Tran-Gia and T. Raith, "Performance analysis of finite capacity polling systems with nonexhaustive service," Performance Evaluation, vol. 9, pp. 1-16, 1988.

[4] M. Lang and M. Bosch, "Performance analysis of finite capacity polling systems with limited-m service," Proc. ITC-13, vol. 14, pp. 731-735, 1991.

[5] P. J. Kuehn, "Multiqueue systems with nonexhaustive cyclic service," B. S. T. J., vol. 58, no. 3, pp. 671-699, 1979.

[6] Fuyung Lai, Performance evaluation of an ATM switch and error control schemes for high speed networks, $\mathrm{PhD}$ thesis, Department of Electrical and Computer Engineering, North Carolina State University, Raleigh, NC, February 1991.

[7] L. Kleinrock, Queueing Systems, Vol. I: Theory. New York, NY: John Wiley \& Sons, Inc, 1975 .

[8] J. J. Hunter, Mathematical Techniques of Applied Probability, Vol. 2, Ch. 9. New York, NY: Academic Press, Inc., 1983.

[9] W. Feller, An Introduction to Probability Theory and Its Applications, Vol. I, 3rd ed. New York, NY: John Wiley \& Sons, Inc, 1968. 
A Refined Approximation of a Finite Capacity Polling System ... Jou, Nilsson, and Lai. 26 [10] Tony T. Lee, "M/G/1/N queue with vacation time and limited service discipline," Performance Evaluation, vol. 9, pp. 181-190, 1989.

[11] E. Gelenbe and I. Mitrani, Analysis and Synthesis of Computer Systems, Ch. 3. New York, NY: Academic Press, Inc., 1980. 